\title{
Energy fluctuations induced by stochastic frequency changes in atom traps
}

\author{
I. Gallardo and R. Jáuregui \\ Instituto de Física, Universidad Nacional Autónoma de México, Apartado Postal 20-364, 01000 México DF, Mexico
}

(Received 5 March 2003; published 22 September 2003)

\begin{abstract}
We study the quantum description of energy fluctuations induced by stochastic changes in the frequency of atom traps. Using the connection between classical and quantum descriptions of parametric oscillators, the classical cumulant expansion method is used to obtain quantum results beyond standard perturbation theory. Both the case of static and time-dependent traps are explicitly worked out.
\end{abstract}

DOI: 10.1103/PhysRevA.68.033807

PACS number(s): 42.50.Ct, 42.50.Lc, 42.50.Dv

\section{INTRODUCTION}

Fluctuations of the electromagnetic fields used to trap or to modify the quantum state of trapped atoms are an important source of decoherence and limit the feasibility of manipulating atomic quantum states. This fact has given a renewed interest in the study of the effects of classical stochastic fields on quantum systems, in particular, the understanding of noise-induced heating in atom traps.

The dynamics of trapped atoms is particularly important both for the understanding of fundamental effects such as the quantum counterparts of chaos [1] and for its applicability in quantum engineering processes such as quantum computing [2]. Noisy electric fields couple to the atom giving rise to a fluctuating interaction. For static traps, fluctuations manifest themselves as either variations on the spring constant or on the equilibrium position. Classically the former corresponds to multiplicative noise while the latter to additive noise. In the quantum-mechanical counterpart, stochastic changes in the equilibrium position can be dealt exactly $[3,4]$ while a perturbative approach is usually employed for fluctuating frequencies $[5,6]$. When fluctuations with zero mean value and with a stationary correlation function are considered, it results that for static traps

(1) Fluctuations in the equilibrium position lead to an increase of the average energy that is quadratic for short times and linear for long times. In the latter case, the energy changes are determined by the spectral density of the fluctuations evaluated at the frequency of the oscillator [3-5,7].

(2) Fluctuations in the frequency of the oscillator lead to an increase of the average energy at a rate that depends on the initial quantum state. For long enough times and initial number states, this rate is determined by the spectral density evaluated at twice the frequency of the oscillator and an asymptotic exponential growth of the energy is expected [5-7].

For radiofrequency traps or static traps subjected to parametric excitation, the confining potential is usually modeled by a harmonic oscillator with a sinusoidally varying frequency. Most studies of energy fluctuations in these traps consider the secular approximation so that the atom is described as an undriven oscillator with an effective frequency. The residual effect of the driving is known as micromotion. A detailed study of micromotion for stochastic variations of the equilibrium position has already been made both perturbatively [6] and nonperturbatively [8]. In this work, we are interested in understanding micromotion effects of stochastic variations of the driven frequency beyond its perturbative description [6]. To that end, we take into account the relationship between classical and quantum descriptions of parametric oscillators. This allows the quantum evaluation of the expectation values of observables such as energy, in terms of solutions of the classical equations of motion [4]. Stochastic fluctuations of the oscillator parameters are incorporated in the classical solutions through the cumulant method and their effect is directly translated to the quantum regime.

In the following section, the relationship between classical and quantum descriptions of a harmonic oscillator with time-dependent frequency is briefly reviewed. Then, the equations of motion for the correlation functions that determine the behavior of an energy operator are established and solved using perturbative and cumulant methods. The case of static and periodic traps are explicitly worked out in Secs. IV and V. Finally, a general discussion of the results is given.

\section{CLASSICAL VERSUS QUANTUM DESCRIPTION OF PARAMETRIC OSCILLATORS}

Let us consider the Hamiltonian

$$
H=\frac{p^{2}}{2 m}+\frac{1}{2} M \omega_{0}^{2}[1-\epsilon(t)] q^{2},
$$

which takes the form

$$
H=\hbar \omega_{0}\left(a^{\dagger} a+1 / 2\right)-\frac{\hbar \omega_{0} \epsilon(t)}{4}\left(a+a^{\dagger}\right)^{2}
$$

when the standard creation $a^{\dagger}$ and annihilation $a$ operators are introduced. The closed nature of the symplectic algebra $\left\{a^{\dagger} a+1 / 2, a^{\dagger 2}, a^{2}\right\}$ under commutation guarantees that the evolution operator can be written in the form

$$
U(t)=e^{c_{0}\left(a^{\dagger} a+1 / 2\right) / 2} e^{c_{-} a^{\dagger 2} / 2} e^{c_{+} a^{2} / 2} .
$$

A connection between the functions $\left\{c_{0}, c_{+}, c_{-}\right\}$and a pair of solutions $h_{1}(t)$ and $h_{2}(t)$ of the classical equations of motion $[9,10]$

$$
\ddot{h}_{k}(t)+[1-\epsilon(t)] h_{k}(t)=0
$$

with the boundary conditions 


$$
\begin{array}{cc}
h_{1}(0)=1, & \dot{h}_{1}(0)=0, \\
h_{2}(0)=0, & \dot{h}_{2}(0)=-1
\end{array}
$$

is explicitly given by

$$
c_{0}=-2 \ln \left(M_{1}\right), \quad c_{+}=M_{2}^{*} / M_{1}, \quad c_{-}=-M_{2} M_{1},
$$

where

$$
\begin{gathered}
M_{1}=\frac{1}{2}\left[\left(h_{1}-i h_{2}\right)-\left(\dot{h}_{2}+i \dot{h}_{1}\right)\right], \\
M_{2}=\frac{1}{2}\left[-\left(h_{1}-i h_{2}\right)-\left(\dot{h}_{2}+i \dot{h}_{1}\right)\right] .
\end{gathered}
$$

In these equations we have used the notation

$$
\dot{f}(t)=\frac{d f}{d \omega_{0} t} .
$$

Consider $\hat{\mathcal{E}}=\hbar \omega_{0}\left(a^{\dagger} a+1 / 2\right)$ as a reference energy operator. Its time evolution can be evaluated directly:

$$
\begin{aligned}
\hat{\mathcal{E}}(t)= & \hbar \omega_{0} \hat{U}^{-1}(t)\left[a^{\dagger} a+1 / 2\right] \hat{U}(t) \\
= & \hbar \omega_{0}\left\{\left(1+2\left|M_{2}\right|^{2}\right)\left(a^{\dagger} a+1 / 2\right)\right. \\
& \left.-M_{1} M_{2} a^{\dagger 2}-M_{1}^{*} M_{2}^{*} a^{2}\right\} .
\end{aligned}
$$

This expression is valid for any realization of $\epsilon(t)$. That is, it admits the possibility that

$$
\begin{aligned}
\varpi_{T} & =: 1-\varepsilon(t) \\
& =\frac{\omega(t)}{\omega_{0}}+\alpha \lambda(t) \\
& =\varpi+\alpha \lambda(t),
\end{aligned}
$$

with $\omega(t)$ deterministic and $\alpha \lambda(t)$ stochastic. The parameter $\alpha$ would then measure the magnitude of the fluctuations.

Equation (9) shows that in order to evaluate the increase of the reference energy due to fluctuations in the frequency of a parametric quantum oscillator it is necessary to study the time evolution of the classical products $h_{i} h_{j}$ that appear in the functions $M_{i} M_{j}$.

\section{A. Stochastic equations of motion of the products $\boldsymbol{h}_{i} \boldsymbol{h}_{j}$}

In the case of products between classical solutions with different initial conditions, define the vector $\mathbf{u}^{(4)}$ with four components:

$$
\begin{aligned}
& u_{1}^{(4)}=h_{1} h_{2}, \\
& u_{2}^{(4)}=\dot{h}_{1} h_{2}, \\
& u_{3}^{(4)}=h_{1} \dot{h}_{2}, \\
& u_{4}^{(4)}=\dot{h}_{1} \dot{h}_{2},
\end{aligned}
$$

Using the classical equation (4) it results that

$$
\begin{gathered}
\dot{u}_{1}^{(4)}=u_{2}^{(4)}+u_{3}^{(4)}, \\
\dot{u}_{2}^{(4)}=-\varpi_{T}^{2} u_{1}^{(4)}+u_{4}^{(4)}, \\
\dot{u}_{3}^{(4)}=-\varpi_{T}^{2} u_{1}^{(4)}+u_{4}^{(4)}, \\
\dot{u}_{4}^{(4)}=-\varpi_{T}^{2} u_{3}^{(4)}+-\varpi_{T}^{2} u_{2}^{(4)},
\end{gathered}
$$

so that

$$
\dot{\mathbf{u}}^{(4)}=\left(\mathbf{A}_{0}^{(4)}+\mathbf{A}_{1}^{(4)}\right) \mathbf{u}^{(4)},
$$

where the nonstochastic matrix $\mathbf{A}_{0}$ is

$$
\mathbf{A}_{0}^{(4)}=\left(\begin{array}{cccc}
0 & 1 & 1 & 0 \\
-\varpi^{2} & 0 & 0 & 1 \\
-\varpi^{2} & 0 & 0 & 1 \\
0 & -\varpi^{2} & -\varpi^{2} & 0
\end{array}\right) .
$$

It can be decomposed in the form

$$
\mathbf{A}_{0}^{(4)}=\mathbf{a}_{0}^{(4)}-\varpi^{2} \mathbf{a}_{0}^{(4) \dagger},
$$

with

$$
\mathbf{a}_{0}^{(4)}=\left(\begin{array}{llll}
0 & 1 & 1 & 0 \\
0 & 0 & 0 & 1 \\
0 & 0 & 0 & 1 \\
0 & 0 & 0 & 0
\end{array}\right),
$$

while the stochastic matrix $\mathbf{A}_{1}$ is given by

$$
\mathbf{A}_{1}^{(4)}(t)=\alpha \lambda(t) \mathbf{a}_{0}^{(4) \dagger} .
$$

Note that in order to close the algebra established by the commutation of $\mathbf{a}_{0}^{(4)}$ and $\mathbf{a}_{0}^{(4) \dagger}$ it is necessary to introduce the matrix $\mathbf{a}_{z}^{(4)}$

$$
\mathbf{a}_{z}^{(4)}=\left(\begin{array}{cccc}
1 & 0 & 0 & 0 \\
0 & 0 & 0 & 0 \\
0 & 0 & 0 & 0 \\
0 & 0 & 0 & -1
\end{array}\right)
$$

so that

$$
\begin{gathered}
{\left[\mathbf{a}_{0}^{(4)}, \mathbf{a}_{0}^{(4) \dagger}\right]=2 \mathbf{a}_{z}^{(4)}, \quad\left[\mathbf{a}_{0}^{(4)}, \mathbf{a}_{z}^{(4)}\right]=-\mathbf{a}_{0}^{(4)},} \\
{\left[\mathbf{a}_{0}^{(4) \dagger}, \mathbf{a}_{z}^{(4)}\right]=\mathbf{a}_{0}^{(4) \dagger} .}
\end{gathered}
$$

For the case of functions $h_{i} h_{i}$ involving the same boundary condition $\mathbf{u}^{(3)}$ is defined by

$$
u_{1}^{(3)}=h_{i} h_{i},
$$




$$
\begin{aligned}
& u_{2}^{(3)}=\dot{h}_{i} h_{i}, \\
& u_{3}^{(3)}=\dot{h}_{i} \dot{h}_{i},
\end{aligned}
$$

with $i=1,2$ and

$$
\begin{gathered}
\dot{u}_{1}^{(3)}=2 u_{2}^{(3)}, \\
\dot{u}_{2}^{(3)}=-\varpi_{T}^{2} u_{1}^{(3)}+u_{3}^{(3)}, \\
\dot{u}_{3}^{(3)}=-2 \varpi_{T}^{2} u_{2}^{(3)} .
\end{gathered}
$$

That is,

$$
\dot{\mathbf{u}}^{(3)}=\left(\mathbf{A}_{0}^{(3)}+\mathbf{A}_{1}^{(3)}\right) \mathbf{u}^{(3)},
$$

where the deterministic matrix is

$$
\mathbf{A}_{0}^{(3)}=\left(\begin{array}{ccc}
0 & 2 & 0 \\
-\varpi^{2} & 0 & 1 \\
0 & -2 \varpi^{2} & 0
\end{array}\right),
$$

so that

$$
\mathbf{A}_{0}^{(3)}=\mathbf{a}_{0}^{(3)}-\boldsymbol{\varpi}^{2} \mathbf{a}_{0}^{(3) \dagger}
$$

with the closed algebra now defined by the matrices

$$
\begin{aligned}
\mathbf{a}_{0}^{(3)} & =\left(\begin{array}{lll}
0 & 2 & 0 \\
0 & 0 & 1 \\
0 & 0 & 0
\end{array}\right), \quad \mathbf{a}_{0}^{(3) \dagger}=\left(\begin{array}{lll}
0 & 0 & 0 \\
1 & 0 & 0 \\
0 & 2 & 0
\end{array}\right), \quad \mathbf{a}_{z}^{(3)} \\
& =\left(\begin{array}{ccc}
1 & 0 & 0 \\
0 & 0 & 0 \\
0 & 0 & -1
\end{array}\right)
\end{aligned}
$$

their commutators are

$$
\begin{gathered}
{\left[\mathbf{a}_{0}^{(3)}, \mathbf{a}_{0}^{(3) \dagger}\right]=2 \mathbf{a}_{z}^{(3)}, \quad\left[\mathbf{a}_{z}^{(3)}, \mathbf{a}_{0}^{(3)}\right]=\mathbf{a}_{0}^{(3)},} \\
{\left[\mathbf{a}_{0}^{(3) \dagger}, \mathbf{a}_{z}^{(3)}\right]=\mathbf{a}_{0}^{(3) \dagger} .}
\end{gathered}
$$

Thus the stochastic matrix can also be written as

$$
\mathbf{A}_{1}^{(3)}(t)=\alpha \lambda(t) \mathbf{a}_{0}^{(3) \dagger} .
$$

Note that $\left\{\mathbf{a}_{0}^{(i)}, \mathbf{a}_{0}^{(i) \dagger}, \mathbf{a}_{z}^{(i)}\right\}, i=3$ and $i=4$, are just three- and four-dimensional matrix realizations of the symplectic algebra $\left\{a^{\dagger 2}, a^{2}, a^{\dagger} a+1 / 2\right\}$.

\section{CUMULANT EXPANSION METHOD}

The deterministic matrix $A_{0}$ can be eliminated from the linear stochastic differential equations (13) and (22) by working in the interaction representation

$$
\mathbf{u}(t)=\mathcal{U} \mathbf{v}(t),
$$

$$
\dot{\mathbf{v}}=\mathcal{U}^{-1} A_{1} \mathcal{U} \mathbf{v}=: \alpha V \mathbf{v}
$$

In general, relevant physical properties will be averaged over a time $T$, which is short compared with the time scale over which the measurable physical quantities change, but large when compared with the correlation time $\tau_{c}$ of the fluctuation. The cumulant expansion [11] for the average $\langle\mathbf{v}(t)\rangle$ is given by

$$
\begin{aligned}
\langle\mathbf{v}(t)\rangle= & \mathcal{T}\left[\operatorname { e x p } \left\{\alpha \int_{0}^{\omega_{0} t} d \omega_{0} t_{1}\left\langle V\left(t_{1}\right)\right\rangle\right.\right. \\
& +\frac{\alpha^{2}}{2} \int_{0}^{\omega_{0} t} d \omega_{0} t_{1} d \omega_{0} t_{2}\left\langle\left\langle V\left(t_{1}\right) V\left(t_{2}\right)\right\rangle\right\rangle+\cdots \\
& +\frac{\alpha^{m}}{m !} \int_{0}^{\omega_{0} t} d \omega_{0} t_{1} d \omega_{0} t_{2} \cdots d \omega_{0} t_{m} \\
& \left.\left.\times\left\langle\left\langle V\left(t_{1}\right) V\left(t_{2}\right) \cdots V\left(t_{m}\right)\right\rangle\right\rangle+\cdots\right\}\right] \mathbf{v}(0) .
\end{aligned}
$$

Standard perturbation theory would approximate

$$
\begin{aligned}
\langle\mathbf{v}(t)\rangle \sim & {\left[1+\alpha \int_{0}^{\omega_{0} t} d \omega_{0} t_{1}\left\langle V\left(t_{1}\right)\right\rangle\right.} \\
& \left.+\frac{\alpha^{2}}{2} \int_{0}^{\omega_{0} t} d \omega_{0} t_{1} d \omega_{0} t_{2}\left\langle\left\langle V\left(t_{1}\right) V\left(t_{2}\right)\right\rangle\right\rangle\right] \mathbf{v}(0)
\end{aligned}
$$

to order $\left(\alpha \omega_{0} t\right)^{2}$. However, being $\tau_{c}$ the correlation time, each cumulant vanishes unless the time points in it are close together within a domain of order $\tau_{c}$. So that the $m$ th term in the exponent in Eq. (29) is of the order of $\alpha \omega_{0} t\left(\alpha \omega_{0} \tau_{c}\right)^{m-1}$, and each term is roughly linear in $t$. The Kubo parameter $\alpha \omega_{0} \tau_{c}$ is determined just by the fluctuations and it will be assumed to be small. This is the advantage of the cumulant expansion as compared with other series expansion in powers of $\alpha \omega_{0} t$ that are valid just for small times $t$. If the cumulant expansion is broken after $m=2$ the resulting equation can be written in the differential form

$$
\frac{\partial\langle\mathbf{v}(t)\rangle}{\partial \omega_{0} t}=\alpha\langle V(t)\rangle+\alpha^{2} \int_{0}^{\omega_{0} t} d \omega_{0} t_{1}\left\langle\left\langle V(t) V\left(t_{1}\right)\right\rangle\right\rangle\langle\mathbf{v}(t)\rangle,
$$

and it is valid to order $\left(\alpha \omega_{0} t\right)\left(\alpha \omega_{0} \tau_{c}\right)$. In terms of the original representation

$$
\begin{aligned}
\frac{\partial\langle\mathbf{u}(t)\rangle}{\partial \omega_{0} t}= & {\left[A_{0}+\left\langle A_{1}\right\rangle+\int_{0}^{\omega_{0} t}\left\langle\left\langle A_{1}(t) \mathcal{U}(t) \mathcal{U}^{-1}(t-\tau)\right.\right.\right.} \\
& \left.\left.\left.\times A_{1}(t-\tau)\right\rangle\right\rangle \mathcal{U}(t-\tau) d \omega_{0} \tau\right]\langle\mathbf{u}\rangle
\end{aligned}
$$




\section{A. Interaction picture for the products $\boldsymbol{h}_{i} \boldsymbol{h}_{j}$}

In order to isolate the effects of the stochastic matrices $A_{1}$ the interaction picture has to be built explicitly. Consider the matrix $\mathcal{U}(0, t)=\mathcal{U}(t)$ that satisfies the equations

$$
\dot{\mathcal{U}}=A_{0} \mathcal{U}, \quad \mathcal{U}(0)=1
$$

Using the fact that $\left\{a_{0}, a_{0}^{\dagger}, a_{z}\right\}$ defines a closed algebra

$$
\mathcal{U}=e^{-\zeta_{1} \mathbf{a}_{0}^{\dagger}} e^{-\zeta_{0} \mathbf{a}_{0}} e^{-\zeta_{z} \mathbf{a}_{z}}
$$

with the initial conditions

$$
\zeta_{0}(0)=\zeta_{1}(0)=\zeta_{z}(0)=0 \text {. }
$$

A direct calculation shows that

$$
\zeta_{0}=g_{1} g_{2}, \quad \zeta_{1}=-\frac{\dot{g}_{1}}{g_{1}}, \quad \zeta_{z}=-2 \ln g_{1},
$$

with

$$
\ddot{g}_{i}+\varpi^{2} g_{i}=0, \quad i=1,2,
$$

solutions of the classical equation of the harmonic oscillator in the absence of fluctuations. The proper initial conditions are

$$
\begin{array}{cc}
g_{1}(0)=1, & \dot{g}_{1}(0)=0, \\
g_{2}(0)=0, & \dot{g}_{2}(0)=-1 .
\end{array}
$$

Note that, as could be expected, the expressions for $\zeta_{0}, \zeta_{1}$, and $\zeta_{z}$, Eqs. (36), are similar to the equations for $c_{+}, c_{-}$, and $c_{0}$, Eqs. (6). It is also direct to write the evolution operator in its matrix form explicitly:

$$
\mathcal{U}_{4 \times 4}(t)=\left(\begin{array}{cccc}
g_{1}^{2} & -g_{1} g_{2} & -g_{1} g_{2} & g_{2}^{2} \\
g_{1} \dot{g}_{1} & -g_{1} \dot{g}_{2} & -g_{2} \dot{g}_{1} & \dot{g}_{2} g_{2} \\
g_{1} \dot{g}_{1} & -g_{2} \dot{g}_{1} & -g_{1} \dot{g}_{2} & \dot{g}_{2} g_{2} \\
\dot{g}_{1}^{2} & -\dot{g}_{1} \dot{g}_{2} & -\dot{g}_{1} \dot{g}_{2} & \dot{g}_{2}^{2}
\end{array}\right)
$$

in the four-dimensional problem, and

$$
\mathcal{U}_{3 \times 3}(t)=\left(\begin{array}{ccc}
g_{1}^{2} & -2 g_{1} g_{2} & g_{2}^{2} \\
g_{1} \dot{g}_{1} & -g_{1} \dot{g}_{2}-g_{2} \dot{g}_{1} & \dot{g}_{2} g_{2} \\
\dot{g}_{1}^{2} & -2 \dot{g}_{1} \dot{g}_{2} & \dot{g}_{2}^{2}
\end{array}\right)
$$

in the three-dimensional case [12]. In fact,

$$
\begin{aligned}
\mathcal{U}= & \left(1-\zeta_{1} \mathbf{a}_{0}^{\dagger}+\frac{\zeta_{1}^{2}}{2} \mathbf{a}_{0}^{\dagger 2}\right)\left(1-\zeta_{0} \mathbf{a}_{0}+\frac{\zeta_{0}^{2}}{2} \mathbf{a}_{0}^{\dagger 2}\right)\left[\left(\cosh \zeta_{z}-1\right) \mathbf{a}_{z}^{2 n}\right. \\
& \left.-\sinh \zeta_{z} \mathbf{a}_{z}^{2 n-1}+1\right] .
\end{aligned}
$$

From these expressions for the matrices $\mathcal{U}$ let us find the interaction matrix $V$ in this picture. Explicitly,

$$
\mathbf{V}=\lambda\left[g_{1}^{2} \mathbf{a}_{0}^{\dagger}+g_{1} g_{2} \mathbf{a}_{z}-g_{1}^{2} \mathbf{a}_{0}\right]
$$

\section{B. Evolution of the averages $\langle u\rangle$ to order $m=2$}

Define

$$
\begin{aligned}
I_{l k}^{m n}(t)= & \left(\alpha \omega_{0}\right)^{2} \int_{0}^{t} d t_{1} \int_{0}^{t_{1}} d t_{2}\left\langle\lambda\left(t_{1}\right) \lambda\left(t_{2}\right)\right\rangle g_{l}\left(t_{1}\right) \\
& \times g_{k}\left(t_{1}\right) g_{m}\left(t_{2}\right) g_{n}\left(t_{2}\right)
\end{aligned}
$$

where the indices $l, k, m, n$ can either be 1 or 2 according to the boundary conditions satisfied by $g$, Eqs. (38). Then

$$
\begin{gathered}
\alpha^{2} \int_{0}^{\omega_{0} t} d \omega_{0} t_{2} \int_{0}^{\omega_{0} t_{1}} d \omega_{0} t_{1}\left\langle\left\langle\lambda\left(t_{1}\right) \lambda\left(t_{2}\right)\right\rangle\right\rangle \mathbf{V}\left(t_{1}\right) \mathbf{V}\left(t_{2}\right) \\
=\left(\begin{array}{ccc}
1-2 I_{22}^{11}+4 I_{12}^{12} & -4 I_{12}^{22} & 2 I_{22}^{22} \\
2 I_{11}^{22} & 1-2\left(I_{11}^{22}+I_{22}^{11}\right) & 2 I_{22}^{12} \\
2 I_{11}^{11} & -4 I_{12}^{11} & 1-2 I_{11}^{22}+4 I_{12}^{12}
\end{array}\right)
\end{gathered}
$$

in the three-dimensional case, and

$$
\alpha^{2} \int_{0}^{\omega_{0} t} d \omega_{0} t_{2} \int_{0}^{\omega_{0} t_{1}} d \omega_{0} t_{1}\left\langle\left\langle\lambda\left(t_{1}\right) \lambda\left(t_{2}\right)\right\rangle\right\rangle \mathbf{V}\left(t_{1}\right) \mathbf{V}\left(t_{2}\right)=\left(\begin{array}{cccc}
1-2 I_{22}^{11}+4 I_{12}^{12} & -2 I_{12}^{22} & -2 I_{12}^{22} & 2 I_{22}^{22} \\
2 I_{11}^{22} & 1-I_{11}^{22}-I_{22}^{11} & -I_{11}^{22}-I_{22}^{11} & 2 I_{22}^{12} \\
2 I_{11}^{22} & -I_{11}^{22}-I_{22}^{11} & 1-I_{11}^{22}-I_{22}^{11} & 2 I_{22}^{12} \\
2 I_{11}^{11} & -2 I_{12}^{11} & -2 I_{12}^{11} & 1-2 I_{11}^{22}+4 I_{12}^{12}
\end{array}\right)
$$

in the four-dimensional case. If the statistical average of the noise is zero,

$$
\langle\lambda(t)\rangle=0
$$

the following perturbative expression for the averaged energy is obtained: 


$$
\begin{aligned}
\hat{\mathcal{E}}(t)= & h \omega_{0}\left\{\left(1+2\left|M_{2}\right|^{2}\right)\left(a^{\dagger} a+1 / 2\right)\right. \\
& \left.-M_{1} M_{2} a^{\dagger 2}-M_{1}^{*} M_{2}^{*} a^{2}\right\},
\end{aligned}
$$

with

$$
\begin{aligned}
\left|M_{2}\right|^{2} \sim & -\frac{1}{2}+\frac{1}{4}\left[\left(g_{1}^{2}+\dot{g}_{1}^{2}+g_{2}^{2}+\dot{g}_{2}^{2}\right)-2\left(g_{1}^{2}+\dot{g}_{1}^{2}\right)\left(I_{22}^{11}-2 I_{12}^{12}\right.\right. \\
& \left.-I_{22}^{22}\right)-4\left(g_{1} g_{2}+\dot{g}_{1} \dot{g}_{2}\right)\left(I_{11}^{12}+I_{22}^{12}\right) \\
& \left.-2\left(g_{2}^{2}+\dot{g}_{2}^{2}\right)\left(I_{11}^{22}-2 I_{12}^{12}-I_{11}^{11}\right)\right], \\
M_{1} M_{2} \sim & \frac{1}{4}\left[\left(g_{1}^{2}+\dot{g}_{1}^{2}\right)\left(-1+2 I_{22}^{11}-4 I_{12}^{12}+2 I_{22}^{22}\right)+\left(g_{2}^{2}+\dot{g}_{2}^{2}\right)(1\right. \\
& \left.-2 I_{11}^{11}+4 I_{12}^{12}-2 I_{11}^{22}\right)+4\left(\dot{g}_{1} \dot{g}_{2}+g_{1} g_{2}\right)\left(I_{11}^{12}-I_{22}^{12}\right) \\
& -4 i\left(g_{1}^{2}+\dot{g}_{1}^{2}\right) I_{12}^{22}+\left(g_{2}^{2}+\dot{g}_{2}^{2}\right) I_{12}^{11}+\frac{1}{2}\left(\dot{g}_{1} \dot{g}_{2}+g_{1} g_{2}\right) \\
& \left.\times\left(1-2 I_{11}^{22}-2 I_{22}^{11}\right)\right] .
\end{aligned}
$$

The nonperturbative result requires solving the Eqs. (32) with a given expression of the noise correlation $\left\langle\lambda(t) \lambda\left(t^{\prime}\right)\right\rangle$.

\section{ENERGY FLUCTUATIONS FOR STATIC HARMONIC TRAPS}

For static traps such as Penning traps or deep optical lattices in a first approximation, the potential is harmonic with a constant frequency $\omega_{0}$. Then,

$$
g_{1}=\cos \omega_{0} t, \quad g_{2}=-\sin \omega_{0} t .
$$

The resulting perturbative expression for the energy is simple because in this case

$$
\begin{gathered}
\left|M_{2}\right|^{2} \sim \frac{\alpha^{2}}{2} \int_{0}^{t} d t_{1} \int_{0}^{t_{1}} d t_{2}\left\langle\lambda\left(t_{1}\right) \lambda\left(t_{2}\right)\right\rangle\left[\cos \left(2 \omega_{0}\left(t_{2}-t_{1}\right)\right],\right. \\
M_{1} M_{2} \sim-\frac{\alpha^{2}}{2} \int_{0}^{t} d t_{1} \int_{0}^{t_{1}} d t_{2}\left\langle\lambda\left(t_{1}\right) \lambda\left(t_{2}\right)\right\rangle e^{-2 i \omega_{0} t_{1}} .
\end{gathered}
$$

Let us assume that the noise is stationary,

$$
\left\langle\lambda(t) \lambda\left(t^{\prime}\right)\right\rangle=\Lambda\left(\left|t-t^{\prime}\right|\right) .
$$

From the last three equations a perturbative expression for the derivative of the energy operator $\mathcal{E}^{(P)}$ is obtained as

$$
\begin{aligned}
\dot{\mathcal{E}}^{(P)}(t)= & \hbar \alpha^{2} \omega_{0}^{2}\left[\int_{0}^{t} d \sigma\langle\lambda(t) \lambda(t-\sigma)\rangle \cos \left(2 \omega_{0} \sigma\right)\right]\left[a^{\dagger} a\right. \\
& +1 / 2]+\frac{\hbar \alpha^{2} \omega_{0}^{2}}{2}\left[e^{-2 i \omega_{0} t} \int_{0}^{t} d \sigma\langle\lambda(t) \lambda(t-\sigma)\rangle\right] a^{\dagger 2} \\
& +\frac{\hbar \alpha^{2} \omega_{0}^{2}}{2}\left[e^{2 i \omega_{0} t} \int_{0}^{t} d \sigma\langle\lambda(t) \lambda(t-\sigma)\rangle\right] a^{2}
\end{aligned}
$$

If the observation time $t$ is such that $\tau_{c} \ll t \ll\left(\alpha \omega_{0}\right)^{-1}$ the integrals may be substituted by the corresponding symmetric spectral densities

$$
\mathcal{S}_{\lambda}(\omega)=\alpha^{2} \int_{0}^{\infty} d \tau \cos (\omega \tau)\langle\lambda(t) \lambda(t-\tau)\rangle,
$$

so that

$$
\begin{aligned}
\mathcal{E}^{(P)}(t)= & \hbar \omega_{0}\left[1+\omega_{0} S_{\lambda}\left(2 \omega_{0}\right) t\right]\left[a^{\dagger} a+1 / 2\right] \\
& +\frac{\hbar \omega_{0}^{2}}{2}\left[\frac{1-e^{-2 i \omega_{0} t}}{2 i}\right] S_{\lambda}(0) a^{\dagger 2} \\
& +\frac{\hbar \omega_{0}^{2}}{2}\left[\frac{e^{2 i \omega_{0} t}-1}{2 i}\right] S_{\lambda}(0) a^{2} .
\end{aligned}
$$

This expression is valid to order $\left(\alpha \omega_{0} t\right)^{2}$ and is consistent with the results obtained for the direct perturbative evolution of initial number states of a harmonic oscillator in the presence of noise.

The cumulant expansion result valid to order $\left(\alpha \omega_{0} t\right)$ $\times\left(\alpha \omega_{0} \tau\right)$ is based on the coupled differential equations (32) which can be used to obtain a differential equation for the energy operator. For stationary noise and if $\tau_{c} \ll t$ $\ll\left(\alpha \omega_{0}\right)^{-1}$,

$$
\dot{\mathcal{E}}(t)=S_{\lambda}\left(2 \omega_{0}\right) \mathcal{E}(t)+\frac{S_{\lambda}(0)}{2}\left[e^{-2 i \omega_{0} t} a^{\dagger 2}+e^{2 i \omega_{0} t} a^{2}\right] .
$$

This equation takes into account that the spectral density already involves a factor $\alpha^{2}$. Solving it one obtains

$$
\begin{aligned}
\mathcal{E}(t)= & \hbar \omega_{0} e^{\omega_{0}^{2} S_{\lambda}\left(2 \omega_{0}\right) t}\left(a^{\dagger} a+1 / 2\right) \\
& +\hbar \omega_{0} \frac{\omega_{0} S_{\lambda}(0)}{4 i+2 \omega_{0} S\left(2 \omega_{0}\right)}\left[e^{\omega_{0}^{2} S_{\lambda}\left(2 \omega_{0}\right) t}-e^{-2 i \omega_{0} t}\right] a^{\dagger 2} \\
& +\hbar \omega_{0} \frac{\omega_{0} S_{\lambda}(0)}{-4 i-2 \omega_{0} S\left(2 \omega_{0}\right)}\left[e^{\omega_{0}^{2} S_{\lambda}\left(2 \omega_{0}\right) t}-e^{2 i \omega_{0} t}\right] a^{2} .
\end{aligned}
$$

Thus, an exponential growth of the energy is expected for a system that was initially in a number state,

$$
\frac{d\langle n|\mathcal{E}| n\rangle}{d \omega_{0} t}=S_{\lambda}\left(2 \omega_{0}\right)\langle n|\mathcal{E}| n\rangle
$$

and deviations from this behavior are expected for other initial states.

\section{ENERGY FLUCTUATIONS FOR TRAPS WITH A PERIODIC TIME-DEPENDENT FREQUENCY}

In this section we consider a trap with a frequency depending on time in a harmonic way. This behavior is expected for stationary traps subjected to parametric heating or for radiofrequency traps. In these cases the normalized frequency $\varpi$ can be identified as 


$$
\varpi^{2}(t)=\left(\frac{\Omega^{2}}{2 \omega_{0}}\right)^{2}[a-2 q \cos (\Omega t)] .
$$

The functions $g_{i}, i=1,2$, satisfy the Mathieu equation and can be expressed in terms of Floquet functions:

$$
\begin{aligned}
& g_{1}(t)=A_{1} \sum_{k=-\infty}^{\infty} c_{n} \cos (\mu+k \Omega t), \\
& g_{2}(t)=A_{2} \sum_{k=-\infty}^{\infty} c_{n} \sin (\mu+k \Omega t),
\end{aligned}
$$

with $\mu$ the secular frequency of the system and the constants $A_{1}$ and $A_{2}$ chosen so that $g_{1}$ and $g_{2}$ satisfy the adequate boundary conditions, that is,

$$
\begin{gathered}
A_{1}^{-1}=\sum_{k=-\infty}^{\infty} c_{k}, \\
A_{2}^{-1}=-\sum_{k=-\infty}^{\infty} c_{k} \frac{\mu+k \Omega}{\omega_{0}} .
\end{gathered}
$$

For a stable behavior of the atom in the trap, $\mu$ is a real number.

We choose the frequency scale $\omega_{0}$ so that $\left|A_{1}\right|=\left|A_{2}\right|$, i.e.,

$$
\omega_{0}=\frac{\left|\Sigma c_{k}(\mu+k \Omega)\right|}{\left|\Sigma c_{k}\right|} .
$$

That is, $\omega_{0}$ is chosen as the average of the frequencies involved in the Mathieu functions with a weight $c_{k}$ fixed by the same functions. The perturbative evaluation of the evolution of the reference energy operator $\hat{\mathcal{E}}(t)=\hbar \omega_{0}\left(a^{\dagger} a+1 / 2\right)$ is clumsy but direct. In it, two kinds of terms are present. The first one corresponds to those terms that appear even in the absence of noise $\hat{\mathcal{E}}_{o}(t)$ and those that require its presence $\hat{\mathcal{E}}_{\lambda}(t)$

$$
\hat{\mathcal{E}}(t)=\hat{\mathcal{E}}_{o}(t)+\hat{\mathcal{E}}_{\lambda}(t)
$$

The first one is given by

$$
\hat{\mathcal{E}}_{o}=\hbar \omega_{0}\left[m_{0}\left(a^{\dagger} a+1 / 2\right)+\left(m_{2} a^{2}+m_{2}^{*} a^{2 \dagger}\right)\right]
$$

with

$$
\begin{gathered}
m_{0}=\frac{A_{1}^{2}}{2} \sum_{k, k^{\prime}} c_{k} c_{k^{\prime}} \cos \left(k-k^{\prime}\right) \Omega t\left[1+\frac{\mu+k \Omega}{\omega_{0}} \frac{\mu+k^{\prime} \Omega}{\omega_{0}}\right], \\
m_{2}=\frac{A_{1}^{2}}{4}\left[\left(\sum_{k} c_{k} e^{i(\mu+k \Omega) t}\right)^{2}-\left(\sum_{k} c_{k} \frac{\mu+k \Omega}{\omega_{0}} e^{i(\mu+k \Omega) t}\right)^{2}\right] .
\end{gathered}
$$

The second term involves infinite series of generalized spectral densities and its derivatives:

$$
\begin{aligned}
\hat{\mathcal{E}}_{\lambda}(t)= & h \omega_{0}\left\{\left[g_{1}^{2}+\dot{g}_{1}^{2}+g_{2}^{2}+\dot{g}_{2}^{2}\right]\left|\mathcal{M}_{2}\right| 2\left(a^{\dagger} a+\frac{1}{2}\right)\right. \\
& +\left[g_{1}^{2}+\dot{g}_{1}^{2}-g_{2}^{2}-\dot{g}_{2}^{2}\right] \mathcal{M}_{1} \mathcal{M}_{2}\left(a^{\dagger 2}\right) \\
& \left.+\left[g_{1}^{2}+\dot{g}_{1}^{2}-g_{2}^{2}-\dot{g}_{2}^{2}\right] \mathcal{M}_{1}^{*} \mathcal{M}_{2}^{*}\left(a^{2}\right)\right\},
\end{aligned}
$$

with

$$
\begin{aligned}
\left|\mathcal{M}_{2}\right|^{2}(t)= & \frac{1}{4} t \sum_{l m r k} C_{l m r k} \pi^{2}\left\{\mathcal{S}_{\lambda}(-2 \mu-l-k)\right. \\
& \left.+\mathcal{S}_{\lambda}(2 \mu+l+k)\right\} \delta_{+-+}^{l m r k} \\
\mathcal{M}_{1} \mathcal{M}_{2}(t)= & \frac{1}{2}\left\{\sum _ { l m r k } C _ { l m r k } \left[\frac { \pi ^ { 2 } } { 4 } \left\{t \mathcal{S}_{\lambda}((l-m) \Omega)\right.\right.\right. \\
& \left.+t \mathcal{S}_{\lambda}(2 \mu+(l+m) \Omega)\right\}\left(\delta_{---}^{l r m k}+\delta_{-+-}^{l r m k}\right) \\
& \left.-\mathcal{S}_{\lambda}^{\prime}(2 \mu+(l+m) \Omega) \delta_{+-+}^{l r m k}\right] \\
& -t \sum_{l m r k} C_{l m r k}\left(\frac{i}{2}\right)\left[2 \mathcal { S } _ { \lambda } ( ( l + m ) \Omega ) \left(\delta_{---}^{l r m k}\right.\right. \\
& \left.\left.\left.+\delta_{-+-}^{l r m k}\right)-\mathcal{S}_{\lambda}(2 \mu+(l+m) \Omega) \delta_{+-+}^{l r m k}\right]\right\}
\end{aligned}
$$

In these equations $C_{l m r k}=c_{l} c_{m} c_{r} c_{k}$ and, for instance, $\delta_{+-+}^{l m r k}=\delta((l+m)-(r+k))$; just the resonant terms have been kept and we are also considering times which are long compared to the correlation time and the inverse of the frequencies.

For usual experimental conditions, the factor $q$ that appears in Mathieu equation for $g_{1}$ and $g_{2}$ is much less than one so that

$$
\begin{aligned}
g_{1}(t) \approx A_{1}\left[\cos (\mu t)-\frac{q \Omega}{4}\left(\frac{\cos (\mu+\Omega t)}{2 \mu+\Omega}\right)\right. \\
\left.+\frac{q \Omega}{4}\left(\frac{\cos (\mu-\Omega t)}{2 \mu-\Omega}\right)\right], \\
g_{2}(t) \approx A_{1}\left[\sin (\mu t)-\frac{q \Omega}{4}\left(\frac{\sin (\mu+\Omega t)}{2 \mu+\Omega}\right)\right. \\
\left.+\frac{q \Omega}{4}\left(\frac{\sin (\mu-\Omega t)}{2 \mu-\Omega}\right)\right],
\end{aligned}
$$

with

$$
\mu \approx \frac{|q| \Omega}{2 \sqrt{2}}
$$

$$
\omega_{0} \approx \mu\left[1+\frac{2 q}{2-q^{2}}\right]
$$



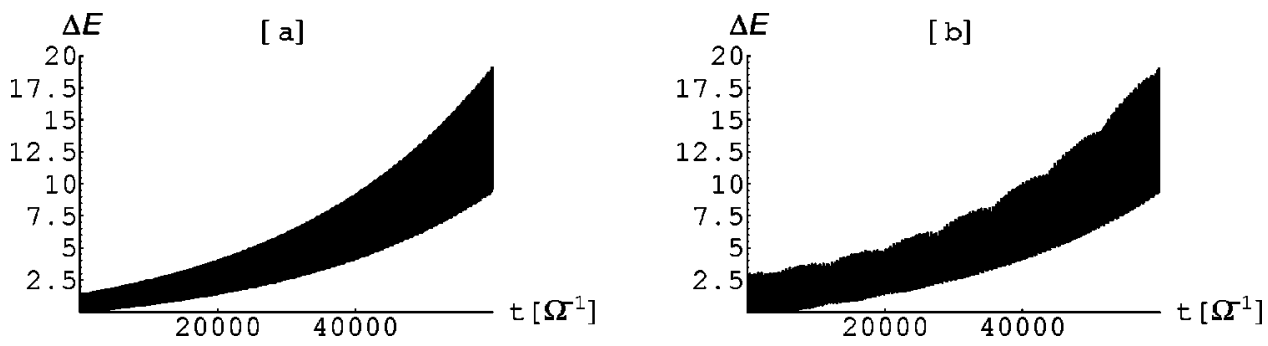

FIG. 1. Evolution of the energy increase $\Delta \mathcal{E}=(\langle\hat{\mathcal{E}}(t)\rangle-\langle\hat{\mathcal{E}}(0)\rangle) / \hbar \omega_{0}$ for the particular case of an initial number state $|n=1\rangle$ (a) and an initial coherent state $|\gamma=1\rangle$ (b) for a trap with parameters $a=0$ and $q=1 / 8$. Time is measured in units of the inverse of the trap frequency $\Omega$; the secular frequency is $\mu=0.045 \Omega$ while the reference frequency is $\omega_{0}=0.051 \Omega$. White noise, $\omega_{\lambda}=0$, a fluctuation intensity $\alpha^{2}$ $=0.01$, and a correlation time $\tau_{c}=0.1 \Omega^{-1}$ are considered.

$$
A_{1}^{-1} \approx\left[1-\frac{q}{2}\right]
$$

The expression for the noise-induced energy changes for number states is then determined by

$$
\begin{aligned}
\left|\mathcal{M}_{2}\right|^{2} \sim & \pi t\left(\frac{2-3 q}{2-q}\right)^{4}\left\{S_{\lambda}(2 \mu)+\frac{2 \mu^{2}}{\Omega^{2}}\left[S_{\lambda}(\Omega+2 \mu)\right.\right. \\
& \left.\left.+S_{\lambda}(\Omega-2 \mu)\right]\right\} .
\end{aligned}
$$

This result is consistent with that obtained semiclassically by Dehmelt [13].

The cumulant method yields results beyond standard perturbation theory. The coupled differential equations (32) can be solved numerically once the correlation function of the noise is specified. In the examples worked out in this paper, the noise is assumed to be stationary, can be colored, and is modeled by

$$
\alpha^{2}\langle\lambda(t) \lambda(t+\sigma)\rangle=\alpha^{2} e^{-|\sigma| / \tau_{c}} \cos \left(\omega_{\lambda} t\right) .
$$

This corresponds to a one-side spectral density of Lorentzian form

$$
S(\omega)=\frac{\alpha^{2} \tau_{c}}{2}\left[\frac{1}{1+\left(\omega_{\lambda}+\omega\right)^{2} \tau_{c}^{2}}+\frac{1}{1+\left(\omega_{\lambda}-\omega\right)^{2} \tau_{c}^{2}}\right]
$$

In Fig. 1, we illustrate the evolution of the energy for the particular case of an initial number state $|n=1\rangle$ and an initial coherent state $|\gamma=1\rangle$. According to Eq. (9) the reference energy would evolve as

$$
\langle n|\hat{\mathcal{E}}(t)| n\rangle=\hbar \omega_{0}\left(1+2\left|M_{2}\right|^{2}\right)[(2 n+1) / 2]
$$

for a given number state and as

$$
\begin{aligned}
\langle\gamma|\hat{\mathcal{E}}(t)| \gamma\rangle= & \hbar \omega_{0}\left\{\left(1+2\left|M_{2}\right|^{2}\right)\left(|\gamma|^{2}+1 / 2\right)-M_{1} M_{2} \gamma^{* 2}\right. \\
& \left.-M_{1}^{*} M_{2}^{*} \gamma^{2}\right\}
\end{aligned}
$$

for a given coherent state. Thus,

$$
\Delta \mathcal{E}_{n}=: \frac{\langle n|\hat{\mathcal{E}}(t)| n\rangle-\langle n|\hat{\mathcal{E}}(0)| n\rangle}{\hbar \omega_{0}}=(2 n+1)\left|M_{2}\right|^{2}
$$

and

$$
\begin{aligned}
\Delta \mathcal{E}_{\gamma}= & \frac{\langle\gamma|\hat{\mathcal{E}}(t)| \gamma\rangle-\langle\gamma|\hat{\mathcal{E}}(0)| \gamma\rangle}{\hbar \omega_{0}}=\left(2|\gamma|^{2}+1 / 2\right)\left|M_{2}\right|^{2} \\
& -M_{1} M_{2} \gamma^{* 2}-M_{1}^{*} M_{2}^{*} \gamma^{2} .
\end{aligned}
$$

In the examples of Fig. 1, we have considered a trap with parameters $a=0$ and $q=1 / 8$ so that the corresponding secular frequency is $\mu=0.045 \Omega$ while the reference frequency is $\omega_{0}=0.051 \Omega$. Noise is taken as white $\left(\omega_{\lambda}=0\right)$ and the fluctuation intensity is $\alpha^{2}=0.01$. The correlation time was taken as $\tau_{c}=0.005 \omega_{0}^{-1}$. In the first graph, it can be observed that $\Delta \mathcal{E}_{n=1}$, and as a consequence the factor $\left|M_{2}\right|^{2}$, exhibits an oscillatory behavior with an amplitude that increases as time increases; an initial linear growth of its average becomes exponential at longer times. This linear growth would also be predicted by perturbation theory for short times. The second graph represents $\Delta \mathcal{E}_{\alpha=1}$ which depends also on $M_{1} M_{2}$. The structure of this term involves oscillations around an average value close to zero for times at which the average value of $\left|M_{2}\right|^{2}$ has become non-negligible. The amplitude of the oscillations is initially $\sim 1.5$ and decreases slowly with time until it becomes almost zero as well as $\operatorname{Re}\left(M_{1} M_{2}\right)$ and $\operatorname{Im}\left(M_{1} M_{2}\right)$. Much later on $\left(t \sim 10^{5} \Omega^{-1}\right)$, the average value of the modulus of $M_{1} M_{2}$ increases exponentially while the amplitude of the oscillations grows. Thus, it can be expected that, in general, the energy fluctuations for a coherent state $\gamma$ will be similar in the average to those of a number state $|n\rangle$ for $t<10^{5} \Omega^{-1}$ whenever $n \sim|\gamma|^{2}$. The main difference between those cases would be the presence of stronger oscillations of the energy at short times for the coherent state than for the number state. This result becomes evident in Fig. 1 for $n=1$ and $\gamma=1$.

We also studied the cases of colored noise with $\omega_{\lambda}=\mu$, $\Omega+\mu$ and $\Omega-\mu$, all other parameters kept identical to those in Fig. 1. For the first case $\left(\omega_{\lambda}=\mu\right)$ the energy increase $\Delta \mathcal{E}$ for number and coherent states is similar to that of Fig. 1, the average energy simply grows with a bigger exponential factor as expected. For $\omega_{\lambda}=\Omega \pm \mu$ the graph of $\left|M_{2}\right|^{2}$ is almost identical to that which gives rise to the results shown in Fig. 
1. The general structure of $M_{1} M_{2}$ is also similar to that illustrated in the figure but the time at which $M_{1} M_{2}$ almost vanishes is shorter. Summarizing, in the case of resonance the evolution of the reference energy for coherent states $\gamma$ and number states $n$ with $|\gamma|^{2}=n$ has a structure similar to the case illustrated in Fig. 1, the most important effect is the faster exponential growth of the mean energy for $\omega_{\lambda}=\mu$.

\section{DISCUSSION}

In this work we have studied the time evolution of harmonic Hamiltonians induced by stochastic changes in the frequency beyond standard perturbation theory. For the case of otherwise static traps, the most important result corresponds to the analytic expression, Eq. (57), which is valid to order $\alpha^{2} \omega_{0}^{2} \tau_{c} t$. The long-time exponential growth predicted by other calculations for number states $[5,6]$ is recovered and extended to any other initial state. At short times, the evolution of number and coherent states is not identical . The evolution of coherent states depends both on the one-side spectral density of the noise at twice the reference frequency $S_{\lambda}\left(2 \omega_{0}\right)$ and its zero frequency value $S_{\lambda}(0)$. It exhibits an oscillatory behavior added to the exponential growth. Meanwhile the energy change for number states is exponential and is determined only by $S_{\lambda}\left(2 \omega_{0}\right)$.

We also studied energy fluctuations for time-dependent traps. It was remarked that the concept of energy is not unique for time-dependent systems. Thus it is important to establish a clear language through, for instance, the definition of a reference Hamiltonian. For periodic variations of the frequency this Hamiltonian is usually taken as representing a harmonic oscillator with a frequency equal to the secular frequency $\mu$. In this work most expressions simplify if we choose a reference oscillator with a frequency $\omega_{0}$ equal to the average defined by Eq. (61). In many practical cases the amplitude of the parametric oscillations is small, that is, $q \ll 1$, and $\omega_{0} \sim \mu$. An alternative corresponds to introduce creation and annihilation operators in an interaction picture so that noise and fluctuation effects are clearly distinguished. This idea is worked out in Ref. [6], where the time evolution of the number operator $A^{\dagger} A$ is studied, $A$ and $A^{\dagger}$ being the invariant operators of the parametric oscillator without noise.

Conversely, the analysis we performed in this paper using a time-independent reference Hamiltonian has a quite clear interpretation. It permits us to separate the energy in the sum of terms due to deterministic and terms due to fluctuating changes in the frequency within the perturbative treatment. Meanwhile the cumulant expansion study not only shows the short-time linear and long-time exponential growth of the average value of the reference energy, but also makes evident that some interesting effects can be expected for nonumber states.

The results of this paper should be useful in the study of the limits of atomic state manipulations set by specific noise sources. It is expected that the noise in a trap depends on its material, geometry, and size, as well as electronic devices. The spectral density of this noise at specific frequencies determines the evolution of the system. Measuring heating rates the values of $S_{\lambda}\left(2 \omega_{0}\right)$ and $S_{\lambda}(0)$ can be inferred. Up to now, most experimental studies in Paul traps concentrate on energy fluctuations starting from the zero number state yielding an important scaling effect within the approximately linear regime $[14,15]$. In order to test the predictions of this work it is necessary to study heating rates for times long enough to observe deviations from the linear behavior and starting from a variety of initial quantum states. For instance, a direct application of Eq. (9) shows that the linear superposition of coherent states $|\gamma\rangle+|-\gamma\rangle$ is heated slower than its counterpart $|\gamma\rangle-|-\gamma\rangle$. Observing the strong oscillations predicted for the expectation value of the reference energy due to micromotion effects would require experimental techniques that lead to measurements of the energy with a high time resolution.
[1] R. Chacón and J.I. Cirac, Phys. Rev. A 51, 4900 (1995); M. El Ghafar, P. Torma, V. Savichev, E. Mayr, A. Zeiler, and W.P. Schleich, Phys. Rev. Lett. 78, 4181 (1997); G.P. Berman, D.F.V. James, R.J. Hughes, M.S. Gulley, M.H. Holzscheiter, and G.V. Lopez, Phys. Rev. A 61, 023403 (2000).

[2] J.I. Cirac and P. Zoller, Phys. Rev. Lett. 74, 4091 (1997); B.E. King, C.S. Wood, C.J. Myatt, Q.A. Turchette, D. Leibfried, W.M. Itano, C. Monroe, and D.J. Wineland, ibid. 81, 1525 (1998); Q.A. Turchette, C.S. Wood, B.E. King, C.J. Myatt, D. Leibfried, W.M. Itano, C. Monroe, and D.J. Wineland, ibid. 81, 3631 (1998).

[3] D.F.V. James, Phys. Rev. Lett. 81, 317 (1998).

[4] R. Jáuregui, Phys. Rev. A 64, 053408 (2001).

[5] T.A. Savard, K.M. O'Hara, and J.E. Thomas, Phys. Rev. A 56, R1095 (1997).

[6] S. Hacyan and R. Jáuregui, J. Opt. B: Quantum Semiclassical Opt. 5, 138 (2003).
[7] S. Schneider and G.J. Milburn, Phys. Rev. A 59, 3766 (1999).

[8] S. Brouard and J. Plata, Phys. Rev. A 63, 043402 (2001).

[9] O. Castaños, R. Jáuregui, R. Lopez-Peña, J. Recamier, and V.I. Man'ko, Phys. Rev. A 55, 1208 (1997).

[10] R. Jáuregui, N. Poli, G. Roati, and G. Modugno, Phys. Rev. A 64, 033403 (2001).

[11] N. G. van Kampen, Stochastic Processes in Physics and Chemistry (North-Holland, Amsterdam, 1981).

[12] I. F. Gallardo, Bachelor thesis, UNAM, 2002 (unpublished).

[13] H.G. Dehmelt, Adv. At. Mol. Phys. 3, 53 (1967); 5, 109 (1967).

[14] Q.A. Turchette, D. Kielpinski, B.E. King, D. Leibfried, D.M. Meekhof, C.J. Myatt, M.A. Rowe, C.A. Sackett, C.S. Wood, W.M. Itano, C. Monroe, and D.J. Wineland, Phys. Rev. A 61, 063418 (2000).

[15] C.F. Roos, D. Leibfried, A. Mundt, F. Schmidt-Kaler, J. Eschner, and R. Blatt, Phys. Rev. Lett. 85, 5547 (2000). 